Proceedings of the International Congress on Advances in Applied Physics and Materials Science, Antalya 2011

\title{
The Smaller, the Better: From the Spider-Spinning to Bubble-Electrospinning
}

\author{
J.-H. HE* \\ National Engineering Laboratory for Modern Silk, College of Textile and Engineering, Soochow University \\ 199 Ren-ai Road, Suzhou 215123, China \\ Nanobubble dynamics in the spider-spinning procedure is elucidated, and a theoretical model is established \\ to show why spider drag-line silk protein assemblies have extraordinary strength and toughness. Suggested by \\ the spider-spinning, a bubble electrospinning is investigated for mass production of nanofibers within diameters \\ $20 \mathrm{~nm}$. El Naschie's E-infinity theory is a universal theory for nanotechnology. It is concluded that smaller is the \\ way to have excellent thermal and electric conductivity, in fact the smaller, the better.
}

PACS: 81.16.-c, 81.07.-b, 81.05.Lg

\section{Introduction}

In the last decade considerable work was invested in developing new methods for producing nanofibers [1-3], and the nanoeffect [4] enables nanofibers to have unusual strength, high surface energy, surface reactivity, high thermal and electric conductivity, these characteristics improve performance for many applications. A spider silk $[5,6]$ is actually a nanofiber assembly consisting of thousands of nanofibers, this distinct character can explain why it is of extraordinary strength and toughness. A possible mechanism in the spider-spinning might be the nanobubble dynamics [7], and the mechanism can be used to produce nanofibers with remarkable strength throughout using polymer bubbles [8-10]. This lecture will also give a brief introduction to $E$-infinity nanoscience and negative space.

\section{Spider-spinning}

Most natural spider silk is only 2.5 to $4 \mu \mathrm{m}$ in diameter. Actually a spider silk is an assembly of nanofibers with diameter of about $20 \mathrm{~nm}$, see Fig. 1. The assembly is the key to explanating its excellent mechanical properties. The number of nanofibers in the assembly can be estimated using the formulation: $n=D^{2} / d^{2}$, where $D$ is diameter of the spider silk, $d$ is the average diameter of a single nanofiber. The mechanical strength of a single nanofiber can be expressed as $\tau=\tau_{0}+k d^{-1 / 2}$, where $k$ is the fitting parameter (material constant), $\tau_{0}$ is the strength of the bulk material. Now considering a micro silk with diameter of $D$ and an assembly with the same diameter consisting of nanofibers with diameter of $d$, we predict $\left(\tau_{\text {dragline }}-\tau_{0}\right) /\left(\tau_{\text {micro }}-\tau_{0}\right)=(D / d)^{5 / 2}$. In case $D=3 \mu \mathrm{m}$ and $d=20 \mathrm{~nm}$, the value is $2.7 \times 10^{5}$. That means the spider silk is 5 orders of magnitude higher than would be predicted from a single homogeneous micro silk. The finding shows it is a challenge

\footnotetext{
* e-mail: hejihuan@suda.edu.cn
}

to develop technologies capable of preparing nanofibers within $20 \mathrm{~nm}$, and the bubble-electrospinning can meet this challenge. Nanobubbles are observed in Fig. 1 during the spider spinning process and bubbles of polymer solutions can be used for nanofiber fabrication with high output [8-10], the minimal diameter reached as small as $20 \mathrm{~nm}[10]$.

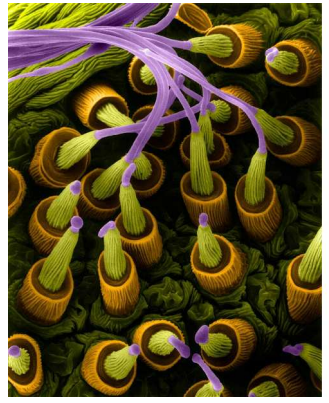

(a)

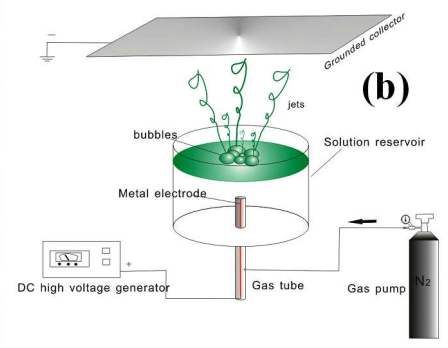

Fig. 1. (a) Spider-spinning process reproduced with the permission of Dennis Kunkel Microscopy, Inc. The diameter of a single nanofiber is about $20 \mathrm{~nm}$. (b) The experimental setup of bubble electrospinning.

\section{Bubble-electrospinning}

We design a new approach, the bubble electrospinning [8-10], to mimick the spider-spinning. The mechanism of the bubble electrospinning process is deceptively simple: in the absence of an electric field, the aerated solution forms various bubbles on the surface. When an electric field is present, it induces charges into the bubble surface, and these quickly relax to the bubble surface. The coupling of surface charge and the external electric field creates a tangential stress, resulting in the deformation of the small bubble into a protuberance-induced upward-directed reentrant jet. Once the electric field exceeds the critical value needed to overcome the surface tension, a fluid jet ejects from the apex of the conical bubble. 
The threshold voltage needed to overcome the surface tension depends upon the size of the bubble and inlet air pressure. The most fascinating character of the surface tension of a bubble is independent of the properties of the electrospun solutions, such as viscosity. This new technology is of critical importance for the new generation of electrospinning, especially for the specialists in design, manufacturing and using nanofibers.

\section{Nanomechanics, is it chaotic or deterministic?}

In view of el Naschie $E$-infinite theory [11], processes at the nanoscale may possess entirely new physical and chemical characteristics that result in properties that are neither well described by those of a single elementary particle of the substance, nor by those of the bulk material. In nanoscale, quantum-like phenomena occur. According to El Naschie [12, 13], nanotechnology is defined as a technology applied in the grey area between classical mechanics and quantum mechanics. Classical mechanics is the mechanics governing the motion of all the objects we can see with our naked eye. This is a mechanics which obeys deterministic laws (Newton laws) and which we can control to a very far extent. By contrast, quantum mechanics which is the mechanics controlling the motion of things like the electron, the proton, the neutron and the like is completely probabilistic.

Nanotechnology links both deterministic classic mechanics and chaotic quantum mechanics. There ought to be a law controlling the change from a classical object like a stone to a quantum object like an electron. Somewhere between these two scales these changes happen, but this does not happen suddenly. There is a grey area between these two scales which is neither classical nor quantum. As $E$-infinity theory is valid for all scales then it follows that it represents a strong candidate for a theory dealing with this grey area.

\begin{tabular}{|llll|}
\hline Quantum world & & \\
\hline Quantum mechanics & Chaotic & $?$ & Nisible world \\
\hline & $?$ & Newton's mechanics \\
\hline & $?$ & \\
\hline
\end{tabular}

Fig. 2. Nanomechanics is chaotic or deterministic?

\section{E-infinity nanoscience}

El Naschie $E$-infinity theory [11] is valid for all scales. In recent years there has been a flurry of original papers published on the foundation and application of el Naschie $E$-infinity Cantorian spacetime theory. The main application of $E$-infinity theory shows miraculous exactness, especially in predicting the theoretical coupling constants and the mass spectrum of the standard model of elementary particles. $E$-infinity nanomechanics can be powerfully applied to nanofibers [14-17].

In the theory of $n$-dimensional spaces, which we mean by $n$-dimensional is simply assumed that we need $n$ numbers representing $n$ coordinates to fix the position of a point in this space. In our classical space time, these are the familiar triple $x, y$ and $z$; while in relativity we have a fourth coordinate or dimension, namely the time $t$. The formal dimension in $E$-infinity theory, however, is $D_{\mathrm{F}}=\infty$. The topological dimension in $E$-infinity theory reads $D_{\mathrm{T}}=4$. The average Hausdorff dimension of El Naschie's spacetime is $D_{\mathrm{H}}=4.23606 \ldots$ That means Einstein's 4-dimensional spacetime is approximately valid, and we predict that there might exist a plane-like string with 2.23606 dimensions on an atom scale. To elucidate this, we can obtain a general expression for boundary dimensions: $D$ (boundary) $=n-1$, where $n$ is the dimension of the geometrical object for which we would like to know the dimension of its boundary. When $n=3.23606$, we have a plane-like string. We can also extend this formula below a point $D=D(0)-1=0-1=-1$ where a negative dimension is obtained. The procedure can continue and we have a -3 space inside a point [18-22].

In an Euclidean space, the separation between two points is measured by the distance between the two points, and is always positive. In spacetime, the interval between two events can be defined as $s^{2}=\Delta r^{2}-c^{2} \Delta t^{2}$, where $c$ is the speed of light, and $\Delta t$ and $\Delta r$ denote differences of the time and space coordinates, respectively, between the events. A natural question then arises: why does a negative sign appear before the temporal separation? It can be completely explained using the negative space [23].

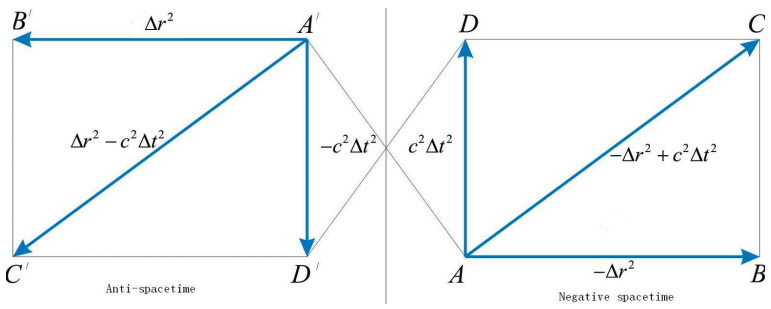

Fig. 3. Negative spacetime beyond the zero point.

The spatial separation in our $3+1$ spacetime is always positive while it becomes negative in a negative space. In a negative spacetime, the interval between two events can be simply obtained using vector addition as illustrated in Fig. 3, that is $-s^{2}=-\Delta r^{2}+c^{2} \Delta t^{2}$; in its anti-space, we recover the general expression.

\section{Acknowledgments}

The work is supported by PAPD (a project funded by the Priority Academic Program Development of Jiangsu Higher Education Institutions), National Natural Sci- 
ence Foundation of China under grant Nos. 10972053, 10802021 , and 50806011.

\section{References}

[1] J.H. He, Polym. Int. 56, 1321 (2007).

[2] J.H. He, Mater. Sci. Tech. 26, 1273 (2010).

[3] J.H. He, Y. Liu, L. Xu, Mater. Sci. Tech. 26, 1275 (2010).

[4] J.H. He, Y.Q. Wan, L. Xu, Chaos Solitons Fract. 33, 26 (2007).

[5] F. Vollrath, Curr. Biol. 16, R925 (2006).

[6] F. Vollrath, D.P. Knight, Nature 410, 541 (2001).

[7] J.H. He, J. Animal Veterin. Adv. 7, 207 (2008).

[8] J.H. He, Y. Liu, L. Xu, J.-Y. Yu, G. Sun, Chaos Solitons Fract. 37, 643 (2008).

[9] Y. Liu, J.H. He, Int. J. Nonlinear Sci. Num. 8, 393 (2007).

[10] R.R. Yang, J.H. He, J.Y. Yu, L. Xu, Int. J. Nonlinear Sci. Num. 11, 163 (2010).

[11] M.S. El Naschie, Chaos Solitons Fract. 19, 209 (2004).
[12] M.S. El Naschie, Chaos, Solitons Fractals 30, 769 (2006).

[13] M.S. El Naschie, Chaos Solitons Fract. 41, 2635 (2009).

[14] J.H. He, Z.F. Ren, J. Fan, L. Xu, Chaos Solitons Fract. 41, 1839 (2009).

[15] J.H. He, Y. Liu, J. Polym. Eng. 28, 101 (2008).

[16] J.H. He, Int. J. Nonlinear Sci. Num. 11, 555 (2010).

[17] J.H. He, Y. Liu, L.-F. Mo, Y.-Q. Wan, L. Xu, Ele ctrospun Nanofibres and Their Applications, Smithers Rapra Update, Shawbury (UK) 2008.

[18] M.S. El Naschie, Nonlinear Sci. Lett. A 2, 1 (2011).

[19] M.S. El Naschie, Nonlinear Sci. Lett. B 1, 4 (2011).

[20] J.-H. He, T. Zhong, L. Xu, L. Marek-Crnjac, S.I. Nada, M.A. Helal, Nonlinear Sci. Lett. B 1, 15 (2011).

[21] L. Marek-Crnjac, Nonlinear Sci. Lett. B 1, 12 (2011).

[22] J.H. He, Nonlinear Sci. Lett. B 1, 6 (2011).

[23] J.H. He, Int. J. Nonlinear Sci. Num. 11, 1093 (2010). 